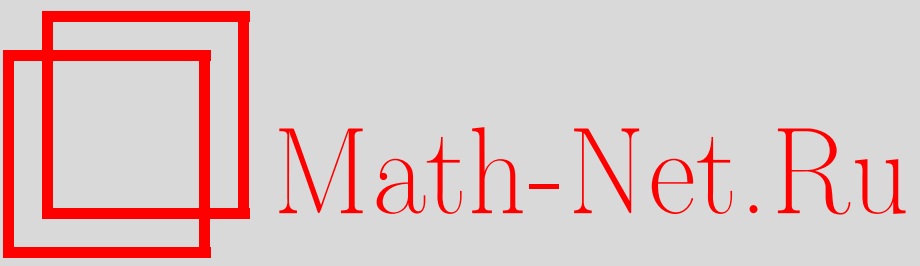

Д. И. Панюшев, О двойных конусах и разложении тензорных произведений, УМH, 1996, том 51, выпуск 2, 171-172

DOI: https://doi.org/10.4213/rm961

Использование Общероссийского математического портала Math-Net.Ru подразумевает, что вы прочитали и согласны с пользовательским соглашением

http://www . mathnet.ru/rus/agreement

Параметры загрузки:

IP : 3.85 .73 .92

26 апреля 2023 г., $15: 25: 51$ 


\title{
О ДВОЙНЫХ КОНУСАХ И РАЗЛОЖЕНИИ ТЕНЗОРНЫХ ПРОИЗВЕДЕНИЙ
}

\author{
Д. И. ПАНЮШЕВ
}

В настоящей заметке излагаются некоторые результаты об алгебрах инвариантов двойных конусов и их приложения к разложению тензорных произведений неприводимых представлений простых алгебр Ли. Основное поле $k$ алгебраически замкнуто и имеет характеристику 0.

Пусть $G$ - простая алгебраическая группа, $U \subset G$ - максимальная унипотентная подгруппа и $V_{\lambda}$ - неприводимый $G$-модуль со старшим весом $\lambda$. Обозначим через $C(\lambda)$ замыкание орбиты старшего вектора в $V_{\lambda}^{*}$. Это аффинный конус над обобшенным флаговым многообразием. Если $\lambda, \mu$ - два доминантных веса, то $C(\lambda, \mu):=C(\lambda) \times C(\mu)$ называется (афинным) двойным конусом. Как показано в [1], [2], описание алгебры $U$-инвариантов (=ковариантов) $k[C(\lambda, \mu)]^{U}$ тесно связано с нахождением разложения серии тензорных произведений $V_{n \lambda} \otimes V_{m \mu}, n, m \in \mathbb{N}$. Более точно, алгебра $k[C(\lambda, \mu)]^{U}$ полиградуирована и, если $r=\operatorname{rk} G$, то градуирующая полугруппа есть $\mathbb{N}^{r+2}$. Допустим нам известно явное выражение для ряда Пуанкаре этой алгебры в виде рациональной функции. Тогда мы можем найти разложение указанной выше серии тензорных произведений, просто разлагая в ряд известную рациональную функцию.

Далее рассматриваются только двойные конусы, связанные с фундаментальными весами. Геометрически это значит, что $C(\lambda, \mu)$ - факториальное многообразие. Структура алгебры $\mathscr{A}:=k[C(\lambda, \mu)]^{U}$ зависит от числа $\widetilde{c}=\widetilde{c}(\lambda, \mu)$, являющегося сложностью двойного конуса относительно действия группы $\widetilde{G}=G \times\left(k^{*}\right)^{2}$ (см. [2], [3]). Если $\widetilde{c}=0$, то $\mathscr{A}$ - алгебра полиномов, и все такие случаи были описаны П. Литтельманом [1]. Все случаи, для которых $\widetilde{c}=1$, были найдены в [2]. Для них алгебра $\mathscr{A}$ оказалась гиперповерхностью (см. [3, Sect. 3]). Наши новые резултаты касаются случая $\widetilde{c}=2$. нумерация фундаментальных весов такая же, как в [4].

Теорема 1. 1. Следующий список содержит все фундаментальные веса $\varphi_{i}$ простых zpynn такие, что $\widetilde{c}\left(\varphi_{i}, \varphi_{i}\right)=2$ :

$$
\begin{aligned}
& \boldsymbol{B}_{m}, \boldsymbol{C}_{m}(m \geqslant 3), \boldsymbol{D}_{m}(m \geqslant 4)-\varphi_{2} ; \boldsymbol{E}_{6}-\varphi_{6} ; \\
& \boldsymbol{E}_{7}-\varphi_{6} ; \boldsymbol{E}_{8}-\varphi_{1} ; \boldsymbol{F}_{4}-\varphi_{1}, \varphi_{4} ; \boldsymbol{G}_{2}-\varphi_{1}, \varphi_{2} .
\end{aligned}
$$

2. Во всех этих случаях алгебра U-инвариантов является полным пересечением гомологической размерности $\leqslant 2$, причем минимальная система образующих и соотноиения явно указываются.

О доКАЗАТЕльствЕ. Список весов извлекается из таблиц в [2]. Для выгисления алгебры $U$-инвариантов используется теорема об ограничении (см. [3, Sect. 1]). При этом выясняется, что почти все двойные конусы из списка имеют изоморфные (или "близкие" ) алгебры $U$-инвариантов.

Заметим, что если старший корень $\Lambda$ простой алгебры Ли фундаментален, то он появляется в вышеприведенном списке. В следующей теореме собраны результаты, относящиеся к этой группе двойных конусов.

Теорема 2. Пусть $G$ - простая алгебраическая группа, такая что $\mathrm{rk} G \geqslant 4$ и $\Lambda$ является фундаментальным весом. Тогда $k[C(\Lambda, \Lambda)]^{U}$ изоморфно некоторой явно указываемой подалгебре в $k\left[C\left(\varphi_{2}, \varphi_{2}\right)\right]^{U\left(D_{4}\right)}=\mathscr{A}$. Более точно,

(1) $k\left[C\left(\varphi_{2}, \varphi_{2}\right)\right]^{U\left(D_{m}\right)} \simeq \mathscr{A} \quad(m \geqslant 4)$, и гомологическая размерность этих алгебр равна 2 ;

(2) существует подалгебра $\mathscr{A}_{1} \subset \mathscr{A}$, имеющая гомологическую размерность 2 такал, что $k\left[C\left(\varphi_{2}, \varphi_{2}\right)\right]^{U\left(B_{m}\right)} \simeq \mathscr{A}_{1}$ nри $m \geqslant 4$

(3) существует подалгебра $\mathscr{A}_{2} \subset \mathscr{A}$, являющаяся гиперповерхностью такая, что $k[C(\Lambda, \Lambda)]^{U} \simeq \mathscr{A}_{2}$ для всех особъц простых групп.

Работа выполнена при финансовой поддержке Российского фонда фундаментальных исследований (грант № 95-01-00783a). 
ПримеР. Опишем строение алгебры $\mathscr{A}:=k\left[C\left(\varphi_{6}, \varphi_{6}\right)\right]^{U\left(E_{6}\right)}$. Будем задавать образующие, выписывая их степени по каждому из сомножителей двойного конуса и соответствующие веса. Эта запись корректна, ибо в каждом таком случае соответствующее весовое подпространство алгебры $\mathscr{A}$ одномерно.

Образующие: $x=\left(1,0, \varphi_{6}\right), y=\left(0,1, \varphi_{6}\right), i=(1,1, \underline{0}), m=\left(1,1, \varphi_{6}\right), n=\left(1,1, \varphi_{3}\right)$, $p=\left(1,1, \varphi_{1} \varphi_{5}\right), \alpha=\left(2,1, \varphi_{3}\right), \beta=\left(1,2, \varphi_{3}\right), q=\left(2,2, \varphi_{2} \varphi_{4}\right)$.

Соотношение: $* x \beta+* y \alpha+* m n=0$. Здесь “*” означает ненулевой скаляр, зависящий от нормировки образующих.

Поэтому мы получаем следующую формулу для ряда Пуанкаре $F_{A}\left(t_{1}, \ldots, t_{6}, z_{1}, z_{2}\right)$ алгебры $\mathscr{A}$ : числитель:

$$
1-z_{1}^{2} z_{2}^{2} t_{3} t_{6}
$$

знаменатель:

$\left(1-z_{1} t_{6}\right)\left(1-z_{2} t_{6}\right)\left(1-z_{1} z_{2}\right)\left(1-z_{1} z_{2} t_{6}\right)\left(1-z_{1} z_{2} t_{3}\right)\left(1-z_{1} z_{2} t_{1} t_{5}\right)\left(1-z_{1}^{2} z_{2} t_{3}\right)\left(1-z_{1} z_{2}^{2} t_{3}\right)\left(1-z_{1}^{2} z_{2}^{2} t_{2} t_{4}\right)$.

Коэффициент при $z_{1}^{n} z_{2}^{m}$ в тейлоровском разложении этой функции (являющийся полиномом от $t_{i}-x$ ) определяет разложение тензорного произведения $V_{n \varphi_{6}} \otimes V_{m \varphi_{6}}$. А именно, моном

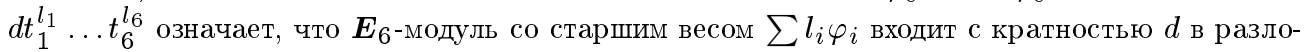
жение этого тензорного произведения.

\section{СПИСОК ЛИТЕРАТУРЫ}

[1] Littelmann P. // J. Algebra. 1994. V. 166. P. 142-157. [2] Panyushev D. // Comment. Math. Helv. 1993. V. 68. P. 455-468. [3] Panyushev D. // Math. Ann. 1995. V. 301. P. 655-675. [4] Винберг Э. Б., Онищик А. Л. Семинар по группам Ли и алгебраическим группам. М.: Наука, 1988. 